\title{
Long Term Medical Treatment of Congenital Hyperinsulinemic Hypoglycemia
}

\author{
Noman Ahmad ${ }^{1 *}$, Mohamad Sharkia ${ }^{2}$ and Laura Stewart ${ }^{3}$ \\ ${ }^{1}$ Department of Endocrinology, King Faisal Specialist Hospital and Research Centre Jeddah, Saudi Arabia \\ ${ }^{2}$ Department of Paediatric Endocrinology, British Columbia Children Hospital Vancouver, Canada \\ ${ }^{3}$ Department of Paediatrics, University of British Columbia, Consultant Paediatric Endocrinologist British Columbia Children \\ Hospital Vancouver, Canada
}

Received: February 24, 2014; Accepted: April 23, 2014; Published: April 24, 2014

*Corresponding author: Noman Ahmad, Consultant Paediatric Endocrinologist, King Faisal Specialist Hospital and Research Centre Jeddah, Saudi Arabia, Tel: +966547660605; E-mail: nomanahmad3@gmail.com

\begin{abstract}
Background: Hyperinsulinism is the most common cause of recurrent Hypoglycemia in early infancy. Surgical treatment with partial/near total pancreatectomy has been the mainstay of treatment but does not result in complete remission of Hypoglycemia and is associated with high risk of diabetes mellitus

Design and methods: We retrospectively reviewed 23 patients (1979-2009) with congenital hyperinsulinism treated medically and surgically. Patients are divided in three groups: 1) treated with diazoxide 2) treated with octreotide alone or along with diazoxide 3) treated with surgical resection. Main outcomes measured are prevention of Hypoglycemia, treatment side effects, onset of diabetes mellitus and effects on growth.
\end{abstract}

Results: Eight patients were treated with diazoxide, 10 with octreotide alone or along with diazoxide and 5 had surgical resection. Four patients came off diazoxide between 5.5-10.5 years age, 3 are less than 5 years and still on treatment. Two patients came off octreotide at 5.5 and 7 years and six remain on treatment with their ages between 6 months and 12 years. No patient could come off medical treatment after surgical resection. 2 patients had diabetes in surgical group and none in medical group.

Conclusion: Medical therapy with diazoxide, octreotide, glucagon and extensive feeding plan is an effective treatment for control of Hypoglycemia in congenital hyperinsulinism patients.

Keywords: Congenital hyperinsulinism; Neonatal Hypoglycemia; 18F-Dopa PET scan; Diazoxide; Octreotide; Near total pancreatectomy

\section{Introduction}

Neonatal Hypoglycemia is a clinical challenge for neonatologist as well as for paediatric endocrinologist. hypoglycemia in infants and children can result in seizures, developmental delay and permanent brain damage. Neonatal Hypoglycemia aetiology ranges from simple delay in fasting adaptation to complex endocrine or metabolic diseases.
Congenital hyperinsulinism $(\mathrm{CH})$ is the most common cause of transient and permanent Hypoglycemia of infancy [1].Congenital Hyperinsulinemic Hypoglycemia is a consequence of dysregulated and inappropriate secretion of insulin due to dysfunctional $\mathrm{K}_{\text {ATP }}$ channels. Prevention of hypoglycaemic episodes is the key to avoid long term neurological sequelae associated with congenital hyperinsulinism $(\mathrm{CH})[2-4]$.

Inadequate suppression of insulin causes increase uptake of glucose by insulin sensitive tissues and inhibition of glycogenolysis, gluconeogenesis, lipolysis and ketogenesis [5]. All these process make developing brain vulnerable to irreversible brain injury. The estimated incidence of $\mathrm{CH}$ in general population is $1 / 50,000$ live births, but it is reported higher in parts of Finland and Saudi Arabia (1/2675 with high consanguineous marriages) [6-8].

The aim of management in infants with $\mathrm{CH}$ is to avoid recurrent episodes of Hypoglycemia and to maintain blood glucose level above $70 \mathrm{mg} / \mathrm{dl}$ (3.9 mmol/l). This is critical to protect brain and prevent seizures associated with Hypoglycemia. Our understanding of genetics, histology and pathogenesis is significantly improved but paediatricians are still struggling to find satisfactory treatment option.

High carbohydrate content feed is necessary to maintain normoglycaemia. Addition of raw corn starch and polycose in feed can improve the carbohydrate content [9]. Intensive feeding plan may be achieved by nasogastric or gastrostomy tube [10].

A number of pharmacological agents are being used to achieve normoglycemia in $\mathrm{CH}$ patients. There must be a rationale approach in introduction, increase in dose and change of drugs. Diazoxide is the first line drug introduced in 1965 for treatment of $\mathrm{CH}$ [11]. Diazoxide is given orally and it is always reasonable to proof that child is not responsive to oral therapy. Diazoxide may cause fluid retention that can precipitate cardiac failure. The most common side effect is the stimulation of generalised 
hypertrichosis. The response to diazoxide can be examined by prolong fasting after 5 days; failure to tolerate fasting indicates insensitive $\mathrm{K}_{\mathrm{ATP}}$ channel [10].

Second-line medical therapy for infants unresponsive to diazoxide is octreotide. Octreotide is a long-acting somatostatin analog that inhibits insulin secretion distal to the $\mathrm{K}_{\mathrm{ATP}}$ channel by inducing hyperpolarization of $\beta$-cells, direct inhibition of voltage dependent calcium channels, and more distal events in the insulin secretory pathway [12]. Octreotide is administered either subcutaneously every $6-8 \mathrm{~h}$ or via continuous infusion. Side effects include suppression of growth, steatorrhea, cholelithiasis and abdominal distension [13]. A rare life threatening effect necrotising enteroclitis is recently reported [14].

Glucagon has been used to increase blood glucose levels by stimulation of hepatic glycogenolysis. The rationale of this treatment comes from the observation of an increased glycogen content of the liver when glycogenolysis is inhibited by the inappropriately high insulin levels [15].

Surgical therapy is indicated in infants who cannot be managed medically or who may have a focal $\mathrm{CH}$ lesion that can be surgically cured [10]. The best available imaging technique is ${ }^{18} \mathrm{~F}$-DOPA PET scan. This advance imaging modality can identify $75 \%$ of focal cases and precisely localise the lesion in all cases [16]. Near total pancreatectomy in patients with diffuse disease often fails to cure hypoglycemia and also lead to early diabetes mellitus [17].

\section{Study Method and Subjects}

The aim of this study is to evaluate the response and longterm outcome of pharmacological treatment in children with Hyperinsulinemic Hypoglycemia. Medical treatment may achieve normoglycaemia and complete resolution of hyperinsulinism with no long-term side effects.

A cohort of 23 children (1979-2009) with multi-ethnic background diagnosed with severe persistent Hypoglycemia in British Columbia children hospital is reviewed. Data is collected retrospectively from hospital charts. Patients are divided in 3 groups on the basis of the treatment modality: 1) Treated with diazoxide 2) Treated with octreotide only or octreotide and diazoxide together 3) Surgical resection.

Outcome measures are: effectiveness of therapy in achieving normoglycemia, age of complete resolution, frequency of severe Hypoglycemia requiring hospital admission, and growth parameters. History of epilepsy and any focal neurological deficit is also reviewed.

\section{Results}

Twenty three patients are divided in three groups: Diazoxide sensitive $(n=8)$, Octreotide group $(n=10)$ and Surgical group $(n=5)$. Patients sensitive to diazoxide presented from age of day 1 to 15 months. Fifty percent $(n=4)$ presented with seizures. Gestational age is full term (38-42 weeks) in most of them. Birth weight ranges from 2.7- 4.65 kilograms. Diazoxide treatment is commenced soon after diagnosis and supplemented with extensive feeding plan. Three patients were given Polycose in first year and 6 received corn starch after $1^{\text {st }}$ year. Four patients were able to stop diazoxide after 5 years of age (5.5-10.5) with euglycaemia. Three patients still less than 5 years and one with GLUD1 mutation are continuing on diazoxide. Hypertrichosis is consistent side effect in all patients. No case of diabetes mellitus in this group yet. Three patients were admitted for Hypoglycemia; total number of admissions is four in whole group. Four patients with learning difficulties, epilepsy, ataxia and global developmental delay all were diagnosed late (10 days, 6 months, 14 months and 15 months). Average height is on $67^{\text {th }}$ percentile $\left(10^{\text {th }}-95^{\text {th }}\right)$.

Ten patients were treated either octreotide alone or octreotide and diazoxide. Age of presentation is from day 1 to 4.5 months; 4 of 10 cases presented in first twenty four hours. Seven patients (70\%) presented with seizures. Gestational age is $40-42$ weeks except one who was 36 weeks. Birth weight ranges from 2.72 to 4.96 kilograms. All patients were given a trial of diazoxide as standard practice except in one whose sibling was previously diagnosed with diazoxide resistant hyperinsulinism. Diazoxide was discontinued in three patients because of side effects; raised liver enzymes, fluid retention with CCF and paralytic ileus. Octreotide is commenced either due to non-responsiveness to diazoxide or its side effects. Lypodystrophy at injection sites was noted in two patients. Two patients came off treatment at 5.5 and 7 years of age. One patient came off octreotide at 3.5 years of age but continued on diazoxide. Seven are still on treatment with their ages from 6 months to 12 years. Extensive feeding plan with polycose or corn starch via nasogastric or gastrostomy tube and continuous feeding is instituted. No major side effects are noted. Average height is on $51^{\text {st }}$ percentile $\left(10^{\text {th }}-90^{\text {th }}\right)$, only one patient had height of -2SDS. No case of diabetes mellitus is reported in this group yet. Eight patients were admitted due to Hypoglycemia; total number of admissions was 14 in the group. Three patients have developmental issues; they were diagnosed at 15 hours (global developmental delay), 4 months (ADHD and learning issues) and 7.5 months (ADHD and learning issues).

Five patients were treated with subtotal pancreatectomy. Gestational age in this group is from 34-41 weeks; birth weight was 3.74-4.03 kilograms. Four patients presented with seizures; age of presentation is immediately after birth, one of them stabilised with feed initially but then presented at 6 months. All patients were given diazoxide as first line treatment. One patient was given octreotide prior to surgery. Sub-total pancreatectomy decision was taken clinically due to poor response and burden of medical therapy. There was no pre-operative imaging to localise focal lesion. No patient could come off medical treatment after surgical resection. Three continued on diazoxide and two on octreotide. Three patients were admitted due to Hypoglycemia; total number of admissions was 4in the group.Two patients developed diabetes at 9 and 12.5 years. Two cases were 7.5 and 12.5 years of age at last follow up visit and they have not developed diabetes yet. Two patients had normal development and 2 developed ADHD and learning issues. One patient follow up was lost at 5 years. 


\section{Discussion}

We presented 23 patients in this observational study who were diagnosed with congenital hyperinsulinism and required prolong course of treatment. We treated 18 patients conservatively and 5 had near total pancreatectomy along with medical therapy. The goal of management in $\mathrm{CH}$ patients is to achieve early and stable euglycaemia and a good neurodevelopment with minimal intervention and limiting the short and long term side effects of therapy. We used diazoxide as first line medical therapy and octreotide are given to those who are nonresponsive to diazoxide. Medical therapy is strongly supplemented with extensive feeding plan which include nasogastric or gastrostomy tube and continuous feed if necessary. Carbohydrate contents of feed is increased with polycose and corn starch. Families are provided home and school nursing support. Hypoglycaemia home management plan of sugar water, glucagon and octreotide is given to all carers. Patients who had only medical treatment no one developed diabetes till last follow-up. In surgical resection group $(n=5) 2$ developed diabetes at 9 and 12.5 years.

Surgical treatment has shown promising results in focal lesions. Majority of patients require less than 50\% pancreatectomy. Near total resection for diffuse lesions has not proven to be effective and not without major side effect of insulin deficiency. Leibowtiz G et al. [17] reported 14 patients with $\mathrm{CH}, 8$ were treated with subtotal resection and 6 had medical treatment. Six patients developed diabetes by puberty; all of them from surgical group. Shilyansky J et al. [18] observed failure of controlling Hypoglycemia in 25\% patients undergoing near total resection and two third of them developing diabetes by the age of puberty. Andrew A et al. [10] reported more than $90 \%$ cure for focal $\mathrm{CH}$, they also reported that cases with diffuse lesion and had $90-95 \%$ resection $50 \%$ of them continued to have Hypoglycemia requiring medical treatment, $25 \%$ were controlled without medical treatment and $25 \%$ developed insulin deficiency. The outcome of our surgical group is comparable with these studies as $100 \%$ continued on medical treatment after resection and $40 \%$ developed diabetes.

Neurodevelopmental outcome is difficult to measure and compare because of highly heterogeneous patients in different reported literature as well as in the same series. $26-44 \%$ patients have shown developmental delay in different studies. Menni $F$ et al. [3] noted neonatal onset as a major risk factor for severe psychomotor retardation and epilepsy. Medical treated patients are less severely affected then those treated by surgical resection. Meissner et al. [19] described 114 patients and noted poor psychomotor outcome with infancy onset and 51\% patients with mental retardation had undergone surgery. Steinkrauss et al. [20] reported 68 patients with $\mathrm{CH}$; one third had developmental delay which was more prevalent in surgical group. Mazor et al. [21] studied 21 Ashkenazi children 12 with diffuse and 9 with focal disease; all of them received medical treatment. Most of the developmental problems resolved by school age and all children were enrolled in regular education. No case of diabetes was noted. In our cohort of 23 patients 13(56.5\%) showed normal development among them 4 were treated with diazoxide, 7 with octreotide and 2 had surgical resection. Over all in medical group $61 \%$ (11 out of 18 patients) and in surgical group 40\% (2 out of 5) showed normal development. Children with developmental delay mostly presented with onset of symptoms in infancy. 3 out of 10 children with developmental delay presented on day one and one of them initially stabilised with feed and represented at 6 months. One child presented at day 10 and others were from 4 months to 15 months old at onset of symptoms. Psychomotor delay or epilepsy in these kids may be secondary to unnoticed Hypoglycemia in neonatal period or in first couple of months.

Octreotide suppresses growth hormone secretion and may have effect on final height. A trend of transient decrease in growth reported previously although ultimate height was not affected [9]. Our patients did not show compromise in their heights as shown at last clinic visits. There is some trend of excessive weight gain seen due to extensive feeding plan.

Conservative management of $\mathrm{CH}$ involves a significant burden on family. It has obvious effects on social life and financial means. A good social support and where ever possible home and school nursing care are necessary. Regular blood glucose monitoring with prompt application of Hypoglycemia management plan is challenging as well as stress full for carrier. Most of these children require bolus or continuous nasogastric or gastrostomy tube feeds supplemented with polycose or corn starch. Intermittent illnesses also further complicate the situation. Medical management work load and responsibility would not be suitable for every family. Motivated and sensible parents need to be reassured that gradually this challenge would ease off as severity of disease decreases after first year of life.

In conclusion medical therapy with diazoxide, octreotide, glucagon and extensive feeding plan is an effective treatment for control of Hypoglycemia in $\mathrm{CH}$ patients. Neurodevelopmental outcome is good if prolong episodes of Hypoglycemia can be prevented. Surgical treatment is a good option for focal disease especially with improved imaging techniques in term of ${ }^{18} \mathrm{~F}$-DOPA PET scan. Near total resection in diffuse lesions does not prevent continuing Hypoglycemia and freedom from medical therapy is not possible. Near total resection also bears significant risk of developing diabetes mellitus by the age of puberty.

There are limitations in our study. Data is collected retrospectively from hospital charts. We do not have definite diagnosis of focal or diffuse lesion as imaging was not performed in most of the patients. Genetic mutations are requested with research laboratories but results are not available. Neurodevelopmental outcome is measured by parent's response and assessment at clinic visits rather than by any formal assessment.

\section{References}

1. Palladino AA, Bennett MJ, Stanley CA (2008) Hyperinsulinism in infancy and childhood: when an insulin level is not always enough. Clin Chem 54(2): 256-263.

2. J Cresto, J Abdenur, I Bergada, R Martino (1998) Long-term follow up of persistent Hyperinsulinemic Hypoglycemia of infancy. Arch Dis Child 79(5): 440-444. 
3. Menni F, de Lonlay P, Sevin C, Touati G, Peigne C et al. (2001) Neurologic outcomes of 90 neonates and infants with persistent hyperinsulinemic hypoglycemia. Pediatrics107(3): 476-479.

4. Izumi T, Takeshige H, Arai T, Sugama M, Mizushima M (1997) Prospective study of nesidioblastosis in newborns and infants: hypoglycemic seizures, epileptogenesis and the significance of the C-peptide suppression test in pancreatectomy. Acta Paediatr Jpn 39(1): 10-17.

5. Hussain K, Oliver Blankenstein, Pascale De Lonlay, Henrik T Christesen (2007) Hyperinsulinaemic Hypoglycemia: biochemical basis and the importance of maintaining normoglycaemia during management. Arch Dis Child 92: 568-570.

6. Mathew PM, Young JM, Abu-Osba YK, Mulhern BD, Hammoudi S et al (1988) Persistent neonatal hyperinsulinism. Clin Pediatr 27(3): 148-151.

7. Aynsley-Green A, Hussain K, Hall J, Saudubray JM, Nihoul- Fekete C et al. (2000) Practical management of hyperinsulinism in infancy. Arch Dis Child Fetal Neonatal Ed 82(2): 98-107.

8. Glaser B, Thornton P, Otonkoski T, Junien C, et al. (2000) Genetics of neonatal hyperinsulinism. Arch Dis Child Fetal Neonatal Ed 82(2): 79-86.

9. Glaser B, Hirsch HJ, Landau H (1993) Persistent hyperinsulinaemic hypoglycemia of infancy: long-term octreotide treatment without pancreatectomy. J Pediatr 123(4): 644-650.

10. Palladino AA, Stanley CA (2011) A specialized team approach to diagnosis and medical versus surgical treatment of infants with congenital hyperinsulinism. Semin Pediatr Surg 20(1): 32-37.

11. Stanley CA, Baker L (1976) Hyperinsulinism in infants and children: diagnosis and therapy. Adv Pediatr 23: 315-355.

12. C Kane, K J Lindley, P R Johnson, R F James, P J Milla et al. (1997) Therapy for persistent hyperinsulinemic hypoglycemia of infancy. Understanding the responsiveness of beta cells to diazoxide and somatostatin. J Clin Invest 100(7): 1888-1893.
13. A Aynsley-Green, K Hussain, J Hall, J Saudubray, C Nihoul-Fekete et al. (2000) Practical management of hyperinsulinism in infancy. Arch Dis Child Fetal Neonatal Ed 82(2): 98-107.

14. Laje P, Halaby L, Adzick NS, Stanley CA ( 2010) Necrotizing enterocolitis in neonates receiving octreotide for the management of congenital hyperinsulinism. Pediatr Diabetes 11(2): 142-147.

15. Cederblad F, Ewald U, Gustafsson J (1998) Effect of glucagon on glucose production, lipolysis, and gluconeogenesis in familial hyperinsulinism. Horm Res 50(2): 94-98.

16. Hardy OT, Hernandez-Pampaloni M, Saffer JR, Scheuermann JS, Ernst LM et al. (2007) Accuracy of [18F]-fluorodopa positron emission tomography for diagnosing and localizing focal congenital hyperinsulinism. J Clin Endocrinol Metab 92(12): 4706-4711.

17. Leibowitz G, Glaser B, Higazi AA, Salameh M, Cerasi E et al. (1995) Hyperinsulinemic hypoglycemia of infancy (nesidioblastosis) in clinical remission: high incidence of diabetes mellitus and persistent beta-cell dysfunction at long-term follow-up. J Clin Endocrinol Metab 80(2): 386392.

18. Shilyansky J, Fisher S, Cutz E, Perlman K, Filler RM et al. (1997) Is 95\% pancreatectomy the procedure of choice for treatment of persistent hyperinsulinaemic hypoglycemia of the neonate? J Pediatr Surg 32(2): 342-346.

19. Meissner T, Wendel U, Burgard P, Schaetzle S, Mayatepek E et al. (2003) Long-term follow-up of 114 patients with congenital hyperinsulinism. Eur J of Endocrinol 149(1): 43-51.

20. Steinkrauss L, Lipman TH, Hendell CD, Gerdes M, Thornton PS et al. (2005) Effects of hypoglycemia on developmental outcome in children with congenital hyperinsulinism. J Pediatr Nurs 20(2): 109-118.

21. Mazor-Aronovitch K, Gillis D, Lobel D, Hirsch HJ, Pinhas-Hamiel O et al. (2007) Long-term neurodevelopmental outcome in conservatively treated congenital hyperinsulinism. Eur J Endocrinol 157(4): 491-497. 